\title{
Time for a motor check
}

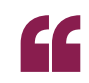

\section{insights \\ into the \\ stator-rotor \\ interaction, stator} assembly and stator maintenance
The bacterial flagellum is a complex nanomachine that is required for motility. The core components of the flagellum are conserved, comprising a hook, a long helical filament and a cell wall-anchored rotary motor, which is composed of stator proteins that surround the rotor. However, previous studies have unravelled species-specific flagellar structures to support various motility modalities and lifestyles.

5 Rotation of the flagellum is driven by stator-rotor interactions coupled to ion or proton flow through a

channel that is in part formed

by transmembrane domains of

the stator; the generated torque on the rotor drives the rotation of the flagellum. However, how ion or proton flow is coupled to mechanical rotation is not fully understood, and detailed structural information about the stator complex and its interaction with the rotor is limited. Two studies now provide structural insights into the stator-rotor interaction, stator assembly and stator maintenance.

Motaleb and Liu laboratories investigated the structure of the motor in the Lyme disease spirochete Borrelia burgdorferi. Stator complexes comprise two transmembrane proteins: MotA and MotB. Here,

the authors showed that compared with wild-type cells, mutants that lacked MotA or MotB had paralysed flagella, which is in agreement with the notion that MotA and MotB form a stator complex necessary for torque generation. MotB contains a conserved aspartate residue in its transmembrane region that has been implicated in proton binding and translocation. Indeed, by introducing amino acid substitutions in MotB, the authors showed that Asp24 is essential for proton translocation, linking proton flux to torque generation by the stator. Imaging analysis revealed that the motor from $\triangle m o t B$ and $\Delta m o t A$ mutants exhibited many features of the wild-type motor, such as the C-ring, the rod and the periplasmic collar (a spirochete-specific structure that has been implicated in recruiting and stabilizing the stator complexes), but lacked 16 transmembrane densities peripheral to the C-ring, which represent MotA-MotB complexes that form the stator ring. Furthermore, the stator complexes interact with the collar, and the periplasmic domains of the 16 stator complexes were inserted between the collar, which might promote stator assembly. Importantly, the stator complex also interacts with the C-ring, which induces a conformational change in the C-ring; as this conformational change is less pronounced in the proton-limited mutant (motB-D24E) and is not observed in the protonblocked mutant (motB-D24N), the authors propose that the protondriven torque is responsible for the conformational change required for flagellar rotation.

Hendrixson and colleagues set out to identify the specific role for FlgX, a protein previously implicated in flagellar motor assembly or function in Campylobacter jejuni. The specific flagellar architecture of C. jejuni is thought to contribute to increased torque and to the higher velocity of motility observed for this bacterium. The authors first showed that although C. jejuni mutants lacking FlgX produced flagella, they exhibited reduced motility compared with the wild type. In addition, they found that FlgX is required for the production of the stator proteins MotA and MotB. Investigating the mechanisms involved in MotA-MotB stability, the authors showed that FlgX does not regulate the MotA-MotB levels at the transcriptional level, thus suggesting that the protein functions post-translationally. Next, they found that FlgX localizes to the cytoplasm and interacts with MotA, possibly via the cytoplasmic domains of MotA; these data support the notion that FlgX maintains the stability of the stator complex. Furthermore, suppressor mutations that reduced the expression of the inner membrane protease $\mathrm{FtsH}$ restored MotA and MotB levels and motility in FlgX-deficient mutants. The findings led the author to hypothesize that FlgX functions as a chaperone that protects stator units from FtsH-mediated proteolysis, thus ensuring that the required torque for flagellar rotation is maintained.

In sum, these studies emphasize the intricate details of the bacterial flagellar motor and strategies to sustain power supply for flagellar rotation.

Andrea Du Toit

ORIGINAL ARTICLES Chang, Y. et al. Structural insights into flagellar stator-rotor interactions. eLife 8, e48979 (2019) | Ribardo, D. A. et al. A chaperone for the stator units of a bacterial flagellum. mBio https://doi.org/10.1128/ mBio.01732-19 (2019) 